\title{
The role of triggers in database forensics
}

\author{
Werner K. Hauger ${ }^{1}$ and Martin S. Olivier ${ }^{2}$ \\ Computer Science Department \\ University of Pretoria \\ Pretoria, South Africa \\ 1'whauger@gmail.com \\ 2 molivier@cs.up.ac.za
}

\begin{abstract}
An aspect of database forensics that has not received much attention in the academic research community yet is the presence of database triggers. Database triggers and their implementations have not yet been thoroughly analysed to establish what possible impact they could have on digital forensic analysis methods and processes. Conventional database triggers are defined to perform automatic actions based on changes in the database. These changes can be on the data level or the data definition level. Digital forensic investigators might thus feel that database triggers do not have an impact on their work. They are simply interrogating the data and metadata without making any changes. This paper attempts to establish if the presence of triggers in a database could potentially disrupt, manipulate or even thwart forensic investigations. The database triggers as defined in the SQL standard were studied together with a number of database trigger implementations. This was done in order to establish what aspects might have an impact on digital forensic analysis. It is demonstrated in this paper that some of the current database forensic analysis methods are impacted by the possible presence of certain types of triggers in a database. Furthermore, it finds that the forensic interpretation and attribution processes should be extended to include the handling and analysis of database triggers if they are present in a database.
\end{abstract}

Keywords-database forensics; database triggers; digital forensic analysis; methods; processes

\section{INTRODUCTION}

Forensic science, or simply forensics, is today widely used by law enforcement to aid them in their investigations of crimes committed. Forensic science technicians, which are specifically trained law enforcement officials, perform a number of forensically sound steps in the execution of their duties. These steps include the identification, collection, preservation and analysis of physical artefacts and the reporting of results. One critical part is the collection and preservation of physical artefacts. The collection needs to be performed in such a manner that the artefacts are not contaminated. The artefacts then need to be preserved in such a way that their integrity is maintained. The reason why this part is so critical is so that any evidence gained from the analysis of these artefacts can not be contested. The evidence found would be used to either implicate or exonerate any involved parties. Any doubt about the integrity of the artefacts collected could lead to the evidence being dismissed or excluded from legal proceedings.
In digital forensics these steps are more commonly referred to as processes. There have been a number of process models developed to guide the digital forensic investigator [1]. The digital forensic process that matches the collection and preservation step in the physical world is the acquisition process. Traditionally, this process involves the making of exact digital copies of all relevant data media identified [19]. However, database forensics needs to be performed on information systems that are becoming increasingly complex. Several factors influence the way that data is forensically acquired and how databases are analysed. They include data context, business continuity, storage architecture, storage size and database models. These factors and their influence on database forensics are examined further in Section II.

Database triggers are designed to perform automatic actions based on events that occur in a database. There is a wide variety of actions that can be performed by triggers. These actions can potentially have an effect on data inside and outside of the DBMS. Thus triggers and the actions they perform are forensically important. This was already recognised by Khanuja and Adane in a framework for database forensic analysis they proposed [4].

The effect that triggers can have on data raises the concern that they could compromise the integrity of the data being investigated. Could triggers due to their nature in combination with the way databases are forensically analysed lead to the contamination of the data that is being analysed? Another concern revolves around the automatic nature of actions performed by triggers. Can the current attribution process correctly identify which party is responsible for which changes?

This paper attempts to establish if these concerns around triggers are justified. The database trigger is defined in the ISO/IEC 9075 SQL standard [5]. Triggers were first introduced in the 1999 version of the standard and subsequently updated in the 2008 version. The specification could thus be examined to determine on a theoretical basis if there is reason for concern. However, the standard is merely used as a guideline by DBMS manufacturers and there is no requirement to conform to the standard. Certain manufacturers also use feature engineering to gain a competitive advantage in the marketplace [6]. They might implement additional triggers based on actual feature requests from high profile clients. Standard triggers might be enhanced or other additional triggers implemented based on 
perceived usefulness by the manufacturers. These features could be used to overcome certain limitations in their DBMS implementations. It is therefore necessary to study actual trigger implementations, rather than the standard itself.

There are thousands of database implementations available and to investigate the trigger implementations of all those databases that use triggers would be prohibitive. Thus, the database trigger implementations of a few proprietary and open-source DBMSs were chosen. The DBMSs investigated were Oracle, Microsoft SQL Server, Mysql, PostgreSQL and DB2. These selected relational database management systems (RDBMS) are widely adopted in the industry. Their dominance in the market means that they would be encountered fairly often by the general digital forensic investigator. These RDBMSs are also the most popular based on the number of web pages on the Internet according to solid IT's ranking method [7]. The official documentation of these RDBMSs was used to study their trigger implementations. The latest published version of the documentation was retrieved from the manufacturer's website [8][9][10][11][12]. At the time of the investigation the latest versions available were as follows: Oracle 11.2g, Microsoft SQL Server 2012, Oracle Mysql 5.7, PostgreSQL 9.3 and IBM DB2 10.

Section II provides the database forensic background against which database triggers will be investigated. Section III describes the database trigger implementations investigated and is divided into four sub-sections: Firstly the triggers defined in the standard were explored. Then the implementations of the standard triggers by the selected DBMSs were examined. Thereafter, other non-standard triggers that some DBMSs have implemented were looked at. For each type of trigger the question was asked as to how the usage of that particular trigger could impact the forensic process or method. Lastly it was established on which objects triggers could be applied. Section IV asks whether the current forensic processes would correctly identify and attribute actions if triggers were used by attackers to commit their crimes. Through the use of a few hypothetical examples as to how triggers could be used by attackers to commit their crimes, this question was investigated. Section V concludes this paper and contemplates further research.

\section{BACKGROUND}

Historically, digital forensics attempts to collect and preserve data media in a static state, which is referred to as dead acquisition [19]. Typically, this process starts with isolating any device that is interacting with a data medium by disconnecting it from all networks and power sources. Then the data medium is disconnected or removed from the device and connected via a write-blocker to a forensic workstation. The write-blocker ensures that the data medium cannot be contaminated while being connected to the forensic workstation. Software is then used to copy the contents to a similar medium or to an alternative medium with enough capacity. Hashing is also performed on the original content with a hash algorithm such as MD5 or SHA-1 [19]. The hashes are used to prove that the copies made are exact copies of the originals and have not been altered. The hashes are also used throughout the analysis process to confirm the integrity of the data being examined. Once the copies have been made, there is no more need for the preservation of the originals [2]. However, if the data being examined is to be used to gather evidence in legal proceedings, some jurisdictions may require that the originals are still available.

A different approach is to perform live acquisition. This involves the collection and preservation of both volatile data (e.g. CPU cache, RAM, network connections) and non-volatile data (e.g. files). Since the acquisition is performed while the system is running, there are some risks that affect the reliability of the acquired data. These risks however can be mitigated by employing certain countermeasures [20].

In today's modern information systems there are several instances where it has become necessary to perform live acquisition. Firstly, in a permanently switched-on and connected world, the context around the imaged data may be required to perform the forensic analysis. This includes volatile items such a running processes, process memory, network connections and logged on users [19]. One area where the context gained from live acquisition is particularly useful is when dealing with possibly encrypted data. This is because the encrypted data might already be open on a running system and the encryption keys used cached in memory [21]. The increasing prevalence of encryption usage to protect data by both individuals and organisations increases the need for more live acquisitions to be performed.

Another instance where live acquisition is performed is when business continuity is required. For many organisations information systems have become a critical part of their operations. The seizure or downtime of such information systems would lead to great financial losses and damaged reputations. The shutdown of mission critical systems might even endanger human life. During forensic investigations, such important information systems can thus no longer be shutdown to perform imaging in the traditional way [19].

The complex storage architecture of today's information systems also necessitates the use of live acquisition techniques. To ensure availability, redundancy, capacity and performance, single storage disks are no longer used for important applications and databases. At least a redundant array of independent disks (RAID) or a full blown storage area network (SAN) is used. Both of these technologies group a variable number of physical storage disks together using different methodologies. They present a logical storage disk to the operating system that is accessible on the block-level.

In such a storage configuration a write-blocker can no longer be efficiently used. There simply may be too many disks in the RAID configuration to make it cost and time effective to image them all [19]. In the case of a SAN, the actual physical disks holding the particular logical disk might not be known, or might be shared among multiple logical disks. These other logical disks may form part of other systems that are unrelated to the application or database system and should preferably not be affected. Attaching the disks in a RAID configuration to another controller with the same configuration can make the data appear corrupt and impossible to access. RAID controller and server manufacturers only support RAID migration 
between specific hardware families and firmware versions. The same would hold true for the imaged disks as well.

While it is still technically possible to image the logical disk the same way as a physical disk, it may not be feasible to do so either. Firstly the size of the logical disk may be bigger than the disk capacity available to the forensic investigator [24]. Secondly the logical disk may hold a lot of other unrelated data, especially in a virtualised environment. Lastly organisations may be running a huge single application or database server containing many different applications and databases. Due to hardware, electricity and licensing costs, the organisation may prefer this to having multiple smaller application or database servers.

Lastly, database systems have their own complexities that affect digital forensic investigations. The models used by the database manufacturers are tightly integrated into their database management systems (DBMS) and are many times of a proprietary nature. Reverse engineering is purposely being made difficult to prevent their intellectual property being used by a competitor. Sometimes reverse engineering is explicitly prohibited in the licensing agreements of the usage of the DBMSs. To forensically analyse the raw data directly is thus not very easy, cost-effective or always possible. The data also needs to be analysed in conjunction with the metadata because the metadata not only describes how to interpret the data, but can also influence the actual seen information [3]. The usage of the DBMS itself, and by extension the model it contains, has become the necessary approach to forensically analyse databases.

The database analysis can be performed in two ways: an analysis on site or an analysis in a clean laboratory environment. On site the analysis is performed on the actual system running the data base. In the laboratory a clean copy of the DBMS with the exact same model as used in the original system is used to analyse the data and metadata acquired [3]. Both ways can be categorised as live analysis due to being performed on a running system. In the first instance the real system is used, while in the second a resuscitated system in a more controlled environment is used e.g. single user, no network connection.

Due to all these complexities associated with applications and particularly databases, live acquisition is the favoured approach when dealing with an information system of a particular size and importance. Fowler documents such a live acquisition in a real world forensic investigation he performed on a Microsoft SQL Server 2005 database [23]. It should be noted that both the operating system and the DBMS are used to access and acquire data after being authenticated. To preserve the integrity of the acquired data, he uses his own clean tools that are stored on a read-only medium [20]. However, the mere accessing of the system will already cause changes to the data, thus effectively contaminating it before it can be copied. Since all the operations performed during the acquisition are documented, they can be accounted for during a subsequent analysis. Hence, this kind of contamination is acceptable as it can be negated during analysis.
Against this background of how forensic acquisition and analysis is performed on a database system, triggers are examined.

\section{TRIGGER IMPLEMENTATION}

This section firstly examines what types of triggers are defined in the standard and how they have been implemented in the DBMSs surveyed. It then looks at other types of triggers that some DBMSs have implemented. Lastly, the database objects that triggers can be applied to, are examined. Throughout the section, the possible impact on database forensics is explored.

\section{A. Definition}

The ISO/IEC 9075 standard part 2: Foundation defines a trigger as an action or multiple actions taking place as a result of an operation being performed on a certain object. The operations are defined as being changes made to rows by inserting, updating or deleting them. Therefore three trigger types are being defined: the insert trigger, the delete trigger and the update trigger. The action can take place immediately before the operation or immediately after the operation. A trigger is thus defined as a BEFORE trigger or an AFTER trigger. The action can take place only once, or it can occur for every row that the operation manipulates. The trigger is thus further defined as a statement-level trigger or as a row-level trigger.

\section{B. Standard triggers}

The first aspect that was looked at was the conformance to the ISO/IEC 9075 SQL standard regarding the type of triggers. All DBMSs surveyed implement the three types of data manipulation language (DML) triggers defined. The only implementations that match the specification exactly in terms of trigger types are those of Oracle and PostgreSQL. They have implemented all combinations of BEFORE/AFTER/Statementlevel/Row-level triggers. The others either place restrictions on the combinations or implement only a subset of the definition from the specification. DB2 has no BEFORE Statement trigger, but all the other combinations are implemented. SQL Server does not implement BEFORE triggers at all. Mysql does not have any statement-level triggers.

Since all three types of DML triggers defined rely on changes of data taking place i.e. either the insertion of new data or the changing or removal of existing data, the standard methods employed by the forensic analyst are not impacted. These methods are specifically chosen because they do not cause any changes and can be used to create proof that in fact no changes have occurred.

Some members of the development community forums have expressed the need for a select trigger [13]. A select trigger would be a trigger that fires when a select operation takes place on the object on which it is defined. None of the DBMSs surveyed implement such a select trigger. Microsoft however is working on such a trigger and its researchers have presented their work already [14]. Oracle on the other hand has created another construct that can be used to perform one of the tasks that the developers want to perform with select triggers: 
manipulate SQL queries that are executed. The construct Oracle has created is called a group policy. It transparently applies the output from a user function to the SQL executed on the defined object for a certain user group. The function can be triggered by selecting, inserting, updating or deleting data. The good news for the forensic analyst is that these functions will not be invoked for users with system privileges. So as long as the forensic analyst uses a database user with the highest privileges, the group policies will not interfere with his investigations.

The existence of a select trigger would have greatly impacted on the standard methods used by the database forensic analyst. One of the methods used to gather data and metadata for analysis is the execution of SQL select statements on system and user database objects such as tables and views. This would have meant that an attacker could have used such a trigger to hide or even worse destroy data. A hacker could use select triggers to booby-tap his root kit. By placing select triggers on sensitive tables used by him, he could initiate the cleanup of incriminating data or even the complete removal of his root kit should somebody become curious about those tables and start investigating.

\section{Non-standard triggers}

The second aspect that was investigated was the additional types of triggers that some DBMSs define. The main reason for the existence of such extra trigger types is to allow developers to build additional and more specialised auditing and authentication functionality, than what is supplied by the DBMS. However that is not the only application area and triggers can be used for a variety of other purposes. For example instead of having an external application monitoring the state of certain elements of the database and performing an action once certain conditions become true, the database itself can initiate these actions.

The non-standard triggers can be categorised into two groups: data definition language (DDL) triggers and other nondata triggers. From the DBMSs investigated, only Oracle and SQL Server provide non-standard triggers.

\section{1) DDL triggers}

The first group of non-standard triggers are the DDL triggers. These are triggers that fire on changes made to the data dictionary with DDL SQL statements e.g. create, drop, alter etc. Different DBMSs define different DDL SQL statements that can trigger actions. SQL Server has a short list that contains just the basic DDL SQL statements. Oracle has a more extensive list and also a special DDL indicator that refers to all of them combined. Since DDL SQL statements can be applied to different types of objects in the data dictionary, these triggers are no longer defined on specific objects. They are rather defined on a global level firing on any occurrence of the event irrespective of the object being changed. Both SQL Server and Oracle allow the scope to be set to a specific schema or the whole database.

These triggers once again rely on data changes being made in the database to fire and thus pose no problem of interference during the forensic investigation.

\section{2) Non-data triggers}

The second group of non-standard triggers are non-data triggers. These are triggers that fire on events that occur during the normal running and usage of a database. Since these triggers do not need any data changes to fire, they potentially have the biggest impact on the methods employed by the forensic analyst. Fortunately the impact is isolated because only a few DBMSs have implemented such triggers.

Both SQL Server and Oracle define a login trigger. This trigger fires when a user logs into the database. SQL Server's login trigger can be defined to perform an action either before or after the login. Authentication however will be performed first in both cases, meaning only authenticated users can activate the trigger. That means the login trigger can be used to perform conditional login or even completely block all logins. An attacker could use this trigger to easily perform a denial of service (DoS) attack. Many applications today use some kind of database connection pool that dynamically grows or shrinks depending on the load of the application. Installing a trigger that prevents further logons to the database would cripple the application during high load. It would be especially bad after an idle period where the application would have reduced its connections to the minimum pool size.

Oracle's login trigger is only performing its action after successful login. Unfortunately that distinction does not make a significant difference and this trigger can also be used to perform conditional login or completely prevent any login. That is because the content of the trigger is executed in the same transaction as the triggering action [16]. Should any error occur in either the triggering action or the trigger itself, then the whole transaction will be rolled back. So simply raising an explicit error in the login trigger will reverse the successful login.

Microsoft has considered the possibility of complete lockout and subsequently created a special method to login to a database that bypasses all triggers. Oracle on the other hand has made the complete transaction rollback not applicable to users with system privileges or the owners of the schemas to prevent a complete lockout. Both SQL Server and Oracle also have a special kind of single-user mode the database can be put into, that will also disable all triggers [15][16].

A hacker could use this trigger to check if a user with system privileges, that has the ability to look past the root kits attempts to hide itself, has logged in. Should such a user log in, he can remove the root kit almost completely, making everything seem normal to the user even on deeper inspection. He can then use Oracle's BEFORE LOGOFF trigger to reinsert the root kit, or use a scheduled task [17] that the root kit hides to re-insert itself after the user with system privileges has logged off.

Another non-data trigger defined by Oracle is the server error trigger. This trigger fires when non-critical server errors occur and could be used to send notifications or perform actions that attempt to solve the indicated error.

The final non-data triggers defined by Oracle only have a database scope due to their nature: the database role change trigger, the database startup trigger and the database shutdown 
trigger. The role change trigger refers to Oracle's proprietary Data Guard product that provides high availability by using multiple database nodes. This trigger could be used to send notifications or to perform configuration changes relating to the node failure and subsequent switch over.

The database startup trigger fires when the database is opened after successfully starting up. This trigger could be used to perform certain initialisation tasks that do not persist and subsequently do not survive a database restart. The database shutdown trigger fires before the database is shut down and could be used to perform cleanup task before shutting down. These last two triggers can be similarly exploited as the login and logoff triggers by a hacker to manage and protect his root kit.

\section{Trigger objects}

The third aspect that was investigated was which database objects the DBMSs allowed to have database triggers. The standard generically defines that triggers should operate on objects, but implies that the objects have rows. It was found that all DBMSs allow triggers to be applied to database tables. Additionally most DBMSs allow triggers to be applied to database views with certain varying restrictions. Only Mysql restricts triggers to be applied to tables only.

None of the DBMSs allow triggers to be applied to system tables and views. Triggers are strictly available only on user tables and views. Additionally there are restrictions to the kind of user table and user views that triggers can be applied to.

This is good news for forensic investigators, since they are very interested in the internal objects that form part of the data dictionary. However there is a move by some DBMSs to provide system procedures and views to display the data from the internal tables [22]. To protect these views and procedures from possible user changes they have been made part of the data dictionary. The ultimate goal seems to be to completely remove direct access to internal tables of the data dictionary.

This might be unsettling news for forensic investigators as they prefer to access any data as directly as possible to ensure the integrity of the data. It will then become important to not only use a clean DBMS, but also a clean data dictionary (at least the system parts). Alternatively the forensic investigator first needs to show that the data dictionary is uncompromised by comparing it to a known clean copy [11]. Only then can he use the functions and procedures provided by the data dictionary.

\section{IDENTITY AND ATTRIBUTION}

The login trigger example brings up another interesting problem. Once the forensic investigator has pieced together all the actions that occurred at the time when the user with system privileges was logged in, he will attribute all the actions to this specific user. This is because all the actions will be tied to him by the audit information. Without looking at triggers, the investigator will miss, that the particular user was completely unaware of certain actions that happened, even though they were triggered and executed with his credentials.
Consider the following example of the salami attack technique: An insurance company pays its brokers commission for each active policy they have sold. The commission amount is calculated according to some formula and the result stored in a commission table with five decimal precision. At the end of the month, a payment process adds all the individual commission amounts together per broker and stores the total amount rounded to two decimals in a payment table. The data from the payment table is then used to create payment instructions for the bank.

Now an attacker could add a BEFORE trigger on the insert/update/delete operations of the commission table which would get executed before the insert/update/delete operation happens. In the trigger, the attacker could truncate the commission amount to two digits; write the truncated portion into the payment table against a dormant broker and the two decimal truncated amounts into the commission table. The banking details of the dormant broker would be changed to an account the attacker controlled and the contact information removed or changed to something invalid so that the real broker would not receive any notification of the payment.

When the forensic investigator gets called in after the fraudulent bank instruction gets discovered, he will find either of two scenarios: The insurance company has an application that uses database user accounts for authentication or an application that has its own built-in authentication mechanism and uses a single database account for all database connections. In the first case, he will discover from the audit logs that possibly all users that have access in the application to manage broker commissions, have at some point updated the fraudulent bank instruction. Surely not all employees are working together to defraud the company. In the second case, the audit logs will attribute all updates to the fraudulent bank instruction to the single account the application uses.

In both cases it would now be worthwhile to query the data dictionary for any triggers that have content that directly or indirectly refers to the payment table. Both Oracle and SQL Server have audit tables that log trigger events. If the trigger events correlate with the updates of the payment table as indicated in the log files, the investigator will have proof that the trigger in fact performed the fraudulent payment instruction updates. He can now move on to determine when and by whom the trigger was created. Should no trigger be found, the investigator can move on to examining the application and its interaction with the database.

Another more prevalent crime that gets a lot of media attention is the stealing of banking details of customers of large companies [18]. The most frequent approach is the breach of the IT infrastructure of the company and the large scale download of customer information including banking details. This normally takes place as a single big operation that gets discovered soon afterwards. A more stealthy approach would be the continuous leaking of small amounts of customer information over a long period.

Triggers could be used quite easily to achieve that at the insurance company in our previous example. The attacker can add an AFTER trigger on the insert/update operations of the banking details table. The trigger takes the new or updated 
banking information and writes it to another table. There might already be such a trigger on the banking details table for auditing purposes and so the attacker simply has to add his part. To prevent any object count auditing picking up his activities, the attacker can use an existing unused table. There is a good chance he will find such a table, because there are always features of the application that the database was designed to have, that simply were not implemented and might never be. This is due to the nature of the dynamic business environment the companies operate in.

Every evening a scheduled task runs that takes all the information stored in the table, puts it in an email and clears the table. There is a possibility that some form of email notification method has already been setup for the database administrator's own auditing process. The attacker simply needs to piggy back on this process and as long as he maintains the same conventions, it will not stand out from the other audit process. Otherwise he can invoke operating system commands from the trigger to transmit the information to the outside. He can connect directly to a server on the Internet and upload the information if the database server has Internet connectivity. Otherwise he can use the email infrastructure of the company to email the information to a mailbox he controls.

The forensic analyst that investigates this data theft will find the same two scenarios as in the previous example. The audit information will point to either of the following: All the staff members are stealing the banking information together or somebody is using the business application to steal the banking details with a malicious piece of functionality. Only by investigating triggers and any interaction with the table that contains the banking information, will he be able to identify the correct party responsible for the data leak.

The actual breach of the IT infrastructure and the subsequent manipulation of the database could have happened weeks or months ago. This creates a problem for the forensic investigator that tries to establish who compromised the database. Some of the log files he normally uses might no longer be available on the system because they have been archived due to space constraints. If the compromise was very far back, some of the archives also might no longer be available because the backup tapes for example might already have been rotated through and reused. The fact that a trigger was used in this example is very useful to the forensic investigator. The creation date and time of trigger can give him a possible beginning for the timeline and more importantly the time window in which the IT infrastructure breach occurred. He can now use the log information he can still get for that time window to determine who is responsible for the data theft.

\section{CONCLUSION AND FUTURE RESEARCH}

Two concerns were raised around the presence of database triggers during forensic investigations. Can triggers cause the contamination of the data being analysed and can the actions performed by triggers be correctly identified and attributed without analysing triggers?

Database triggers are generally defined to perform actions based on changes in the database, be it on the data level or the data definition level. This will normally not affect the work of a forensic analyst, since he is primarily viewing information (be it data or metadata) without making any changes. However some DBMS's allow triggers to be set on the accessing of information. If the forensic analyst works with an Oracle or SQL Server database, he needs to consider the non-data triggers. He should take great care in how he connects to the database to prevent unintended changes from happening and thus potentially having to do time consuming reconstruction to get back to the initial state of the database.

Furthermore triggers can be used to facilitate malicious actions on the back of normal application or operational actions on the database. These changes would be executed in the context of the initial change and the standard audit material would attribute all changes to the same user. It is therefore necessary to examine database triggers as part of the forensic interpretation and attribution processes. All types of triggers should be examined for out of the ordinary and suspicious actions that relate to the compromised data. This is needed to separate the user actions from the automatic trigger actions.

Further research is being conducted to determine how to best analyse the different kinds of triggers. Attention also needs to be given to the fact that some DMBSs allow the obfuscation of the trigger content. An aspect that has not been addressed in this paper is what impact triggers have when the forensic investigator does make intentional changes on a copy of the data. The investigator could be testing a hypothesis, performing data reduction, reconstructing deleted data or simply be storing his results in a temporary table.

\section{REFERENCES}

[1] Pollitt, M.M. (2007). An Ad Hoc Review of Digital Forensic Models. Proceedings of the Second International Workshop on Systematic Approaches to Digital Forensic Engineering. IEEE:43-54.

[2] Cohen, F. (2009). Digital Forensic Evidence Examination - 4th Edition. Livermore, CA.: Fred Cohen \& Associates.

[3] Olivier, M.S. (2009). On metadata context in Database Forensics. Digital Investigation. 5(3-4):115-123.

[4] Khanuja, H.K. \& Adane, D.S. (June 2012). A framework for database forensic analysis. Computer Science \& Engineering 2(3).

[5] ISO/IEC 9075-2. Information technology - Database languages - SQL Part 2:Foundation (SQL/Foundation).

[6] Turner, C.R., Fuggetta, A., Lavazza, L. \& Wolf, A.L. (1999) A conceptual basis for feature engineering. The Journal of Systems and Software 49(1):3-15.

[7] DB-Engines Ranking of Relational DBMS. Available from: http://dbengines.com/en/ranking/relational+dbms (1 May 2014).

[8] CREATE TRIGGER Statement, Oracle® Database PL/SQL Language Reference $11 \mathrm{~g}$ Release 2 (11.2). Available from: http://docs.oracle.com/cd/E11882_01/appdev.112/e17126/create_trigger. htm (2 May 2014).

[9] CREATE TRIGGER, Data Definition Language (DDL) Statements. Available from: http://msdn.microsoft.com/en-us/library/ms189799.aspx (2 May 2014).

[10] CREATE TRIGGER Syntax, MySQL 5.7 Reference Manual. Available from: http://dev.mysql.com/doc/refman/5.7/en/create-trigger.html (2 May 2014).

[11] CREATE TRIGGER, PostgreSQL 9.3.4 Documentation. Available from: http://www.postgresql.org/docs/9.3/static/sql-createtrigger.html (2 May 2014).

[12] CREATE TRIGGER, DB2 reference information. Available from: http://publib.boulder.ibm.com/infocenter/dzichelp/v2r2/index.jsp?topic= 
/com.ibm.db2z10.doc.sqlref/src/tpc/db2z_sql_createtrigger.htm (2 May 2014).

[13] Oracle Developer

Community. https://community.oracle.com/community/developer/search.jspa?people Enabled=true \&userID $=\&$ containerType $=\&$ container $=\& q=$ select + trigger

[14] Fabbri, D., Ramamurthy, R. \& Kaushik, R. (2013). SELECT triggers for data auditing. Proceedings of the 29th International Conference on Data Engineering (ICDE). IEEE:1141-1152

[15] Logon Triggers, Database Engine Instances (SQL Server). Available from: http://technet.microsoft.com/en-us/library/bb326598.aspx (2 May 2014).

[16] PL/SQL Triggers, Oracle ${ }^{\circledR}$ Database PL/SQL Language Reference 11g Release 2 (11.2). Available from: http://docs.oracle.com/cd/E11882_01/appdev.112/e17126/triggers.htm (2 May 2014).

[17] Kornbrust, A. (April 2005). Database rootkits. Presented at Black Hat Europe. Available from: http://www.red-databasesecurity.com/wp/db_rootkits_us.pdf (1 May 2014).

[18] Osborne, C. (13 February 2014). How hackers stole millions of credit card records from Target. ZDNet. Available from: http://www.zdnet.com/how-hackers-stole-millions-of-credit-cardrecords-from-target-7000026299/ (5 May 2014).

[19] Adelstein, F. (February 2006). Live forensics: diagnosing your system without killing it first. Communications of the ACM 49(2):63-66.

[20] Carrier, B.D. (February 2006). Risks of live digital forensic analysis. Communications of the ACM 49(2):56-61.

[21] Hargreaves, C. \& Chivers, H. (2008). Recovery of Encryption Keys from Memory Using a Linear Scan. Proceedings of the Third International Conference on Availability, Reliability and Security (ARES). IEEE:1369-1376.

[22] Lee, M. \& Bieker, G. (2009). Mastering SQL Server 2008. Indianapolis, Indiana: Wiley Publishing Inc.

[23] Fowler, K. (2007). A real world scenario of a SQL Server 2005 database forensics investigation. Available from: https://www.blackhat.com/presentations/bh-usa07/Fowler/Whitepaper/bh-usa-07-fowler-WP.pdf (3 July 2014).

[24] Garfinkel, S.L. (2010). Digital forensics research: The next 10 years. Digital Investigation 7(Supplement):S64-S73. 